\title{
Gas Density Perturbations Induced by One or More Forming Planets in the AS 209 Protoplanetary Disk as Seen with ALMA
}

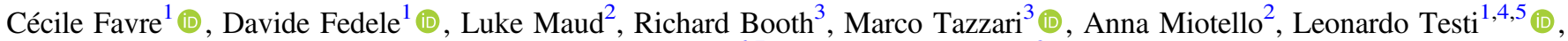 \\ Dmitry Semenov ${ }^{6,7}$, and Simon Bruderer ${ }^{8}$ \\ ${ }^{1}$ INAF-Osservatorio Astrofisico di Arcetri, Largo E. Fermi 5, I-50125, Florence, Italy; cfavre@arcetri.astro.it \\ ${ }^{2}$ Leiden Observatory, Leiden University, P.O. Box 9513, 2300 RA Leiden, The Netherlands \\ ${ }^{3}$ Institute of Astronomy, University of Cambridge, Madingley Road, Cambridge CB3 0HA, UK \\ ${ }^{4}$ European Southern Observatory, Karl-Schwarzschild-Str. 2, D-85748 Garching, Germany \\ ${ }_{5}$ Excellence Cluster Universe, Boltzmannstr. 2, D-85748 Garching, Germany \\ ${ }^{6}$ Max Planck Institute for Astronomy, Knigstuhl 17, D-69117 Heidelberg, Germany \\ ${ }^{7}$ Department of Chemistry, Ludwig Maximilian University, Butenandtstr. 5-13, D-81377 Munich, Germany \\ ${ }^{8}$ Max Planck Institut für Extraterrestrische Physik, Giessenbachstrasse 1, D-85748 Garching, Germany \\ Received 2018 August 2; revised 2018 December 10; accepted 2018 December 10; published 2019 January 25
}

\begin{abstract}
The formation of planets occurs within protoplanetary disks surrounding young stars, resulting in perturbation of the gas and dust surface densities. Here we report the first evidence of spatially resolved gas surface density $\left(\Sigma_{g}\right)$ perturbation toward the AS 209 protoplanetary disk from the optically thin $\mathrm{C}^{18} \mathrm{O}(J=2-1)$ emission. The observations were carried out at $1.3 \mathrm{~mm}$ with Atacama Large Millimeter/submillimeter Array at a spatial resolution of about 0 !" $3 \times 0$ !' 2 (corresponding to $\sim 38 \times 25$ au). The $\mathrm{C}^{18} \mathrm{O}$ emission shows a compact ( $\leqslant 60 \mathrm{au}$ ), centrally peaked emission and an outer ring peaking at $140 \mathrm{au}$, consistent with that observed in the continuum emission, and its azimuthally averaged radial intensity profile presents a deficit that is spatially coincident with the previously reported dust map. This deficit can only be reproduced with our physico-thermochemical disk model by lowering $\Sigma_{\text {gas }}$ by nearly an order of magnitude in the dust gaps. Another salient result is that, contrary to $\mathrm{C}^{18} \mathrm{O}$, the $\mathrm{DCO}^{+}(J=3-2)$ emission peaks between the two dust gaps. We infer that the best scenario to explain our observations $\left(\mathrm{C}^{18} \mathrm{O}\right.$ deficit and $\mathrm{DCO}^{+}$enhancement) is a gas perturbation due to one or more forming planets, which is commensurate with previous continuum observations of the source along with hydrodynamical simulations. Our findings confirm that the previously observed dust gaps are very likely due to perturbation of the gas surface density that is induced by a planet of at least $0.2 M_{\mathrm{J}}$ in formation. Finally, our observations also show the potential of using CO isotopologues to probe the presence of one or more Saturn-mass planets.
\end{abstract}

Key words: ISM: molecules - planet-disk interactions - protoplanetary disks

\section{Introduction}

Formation of planets occurs within the gaseous and dusty interior of protoplanetary disks during the early phase of star formation. Although it remains difficult to directly detect planets in formation, the effect of the planet-disk interaction makes the indirect detection possible via the means of observations of the dust and molecular content of protoplanetary disks. Indeed, while forming, a planet will open gap(s) within the disk, leading to a localized deficit in dust and gas (e.g., Papaloizou \& Lin 1984; Paardekooper \& Mellema 2004; Dong et al. 2015, 2017; Dürmann \& Kley 2015; Rosotti et al. 2016; Bae et al. 2017, 2018). This will result in dust and molecular gaps and rings. In that light, thanks to the high resolution and sensitivity of the Atacama Large Millimeter/ submillimeter Array (ALMA) and the Spectro-Polarimetric High-contrast Exoplanet (SPHERE) facility, such structures have been observed in continuum emission (e.g., ALMA Partnership et al. 2015; Andrews et al. 2016; Isella et al. 2016; Zhang et al. 2016; Fedele et al. 2017, 2018; Loomis et al. 2017; van Boekel et al. 2017; Muro-Arena et al. 2018).

Using ALMA observations, Teague et al. (2017) has reported oscillatory features in CS emission toward TW Hya, and Harsono et al. (2018) have reported a deficit of CO isotopologue emission within 15 au of the disk surrounding TMC-1A. Those deficits might be due not only to grain size and growth properties but also to one ore more planets in formation.

Recently, using ALMA observation of CO, Teague et al. (2018a) and Pinte et al. (2018) claimed the first kinematic evidence of one or more embedded forming planets in the protoplanetary disk surrounding the Herbig Ae star HD 163296. Interestingly enough, hydrodynamical models predict that not only the kinematics but also the bulk of the emission of $\mathrm{CO}$ isotopologues can be used as an indirect probe of the planet-disk interaction (e.g., Ober et al. 2015; Facchini et al. 2018), which induces a gap in the gas at the planet location.

In this paper, we investigate gas perturbations caused by a forming planet in the disk surrounding the T Tauri star AS 209 (Andrews et al. 2009; Huang et al. 2016, 2017; Fedele et al. 2018; Teague et al. 2018b), which is located at $126 \mathrm{pc}$ from the Sun (Gaia Collaboration et al. 2016). For this purpose, we follow up the observations by Fedele et al. (2018, hereafter Paper I), in which the authors inferred that the observed rings and gaps of the $1.3 \mathrm{~mm}$ continuum emission are likely due to the formation of planets; this hypothesis is commensurate with hydrodynamical simulations (for further details, see Paper I). In this study, we only focus on the emission lines of $\mathrm{CO}$ and its isotopologues ${ }^{13} \mathrm{CO}$ and $\mathrm{C}^{18} \mathrm{O}$, along with that of $\mathrm{DCO}^{+}$. In Section 2, we briefly present the observations and the methodology used for combining ALMA observations performed during different cycles. Results and modeling are 
Table 1

Spectroscopic and Observational Line Parameters

\begin{tabular}{|c|c|c|c|c|c|c|c|c|}
\hline \multirow{2}{*}{ Molecule } & \multirow{2}{*}{ Transition } & \multirow{2}{*}{$\begin{array}{l}\text { Frequency } \\
(\mathrm{MHz})\end{array}$} & \multirow{2}{*}{$\begin{array}{l}E_{\mathrm{up}} \\
(\mathrm{K})\end{array}$} & \multirow{2}{*}{$\begin{array}{l}S \mu^{2} \\
\left(\mathrm{D}^{2}\right)\end{array}$} & \multicolumn{2}{|c|}{ Synthesized Beam } & \multirow{2}{*}{$\begin{array}{c}\mathrm{rms} \\
\left(\mathrm{mJy}^{-1} \text { beam }^{-1}\right)\end{array}$} & \multirow{2}{*}{$\begin{array}{l}\text { Integrated Flux }{ }^{\mathrm{a}} \\
\left(\mathrm{K} \mathrm{km} \mathrm{s}^{-1}\right)\end{array}$} \\
\hline & & & & & $(\operatorname{arcsec} \times \operatorname{arcsec})$ & PA (deg) & & \\
\hline $\mathrm{CO}$ & $(2-1)$ & 230538.0 & 16.6 & 0.02 & $0.25 \times 0.21$ & -75.85 & 3.5 & $27.1 \pm 3.9$ \\
\hline${ }^{13} \mathrm{CO}$ & $(2-1)^{b}$ & 220398.7 & 15.9 & 0.05 & $0.25 \times 0.21$ & -73.43 & 3.5 & $8.5 \pm 2.6$ \\
\hline $\mathrm{C}^{18} \mathrm{O}$ & $(2-1)$ & 219560.4 & 15.8 & 0.02 & $0.26 \times 0.22$ & -72.41 & 2.7 & $3.4 \pm 2.1$ \\
\hline $\mathrm{DCO}^{+}$ & $(3-2)^{b}$ & 216112.6 & 20.7 & 142 & $0.26 \times 0.21$ & -73.87 & 2.6 & $2.6 \pm 2.0$ \\
\hline
\end{tabular}

Notes. We used the spectroscopic data parameters from Caselli \& Dore (2005) for DCO ${ }^{+}$and from Winnewisser et al. (1985), Goorvitch (1994), Winnewisser et al. (1997), Cazzoli et al. (2004), and Klapper et al. (2001) for CO and its isotopologues.

${ }^{a}$ Measured disk-averaged line integrated intensity over the line profile using an elliptical aperture of $200 \mathrm{au}$.

${ }^{\mathrm{b}}$ Hyperfine splitting.

presented in Sections 3 and 4, respectively, and a discussion is provided in Section 5.

\section{Observations and Data Reduction}

The observations of the $\mathrm{CO}(2-1),{ }^{13} \mathrm{CO}(2-1), \mathrm{C}^{18} \mathrm{O}(2-1)$, and $\mathrm{DCO}^{+}(3-2)$ lines (see spectroscopic parameters in Table 1) were performed with ALMA toward AS 209 with 38 antennas on 2016 September 22 and 41 antennas on 2016 September 26 (project ID ALMA\#2015.1.00486.S, PI: D. Fedele) toward the following phase-tracking center $\left(\alpha_{\mathrm{J} 2000}=16^{\mathrm{h}} 49^{\mathrm{m}} 15^{\mathrm{s}} .296\right.$, $\delta_{\mathrm{J} 2000}=-14^{\circ} 22^{\prime} 09^{\prime \prime}$.02). The observations cover the frequency range $211-275 \mathrm{GHz}$ in band 6 . For further details, see Paper I.

To optimize the $u v$-coverage together with the sensitivity, we have combined our data with the ones observed by Huang et al. (2016) with ALMA during its Cycle 2 (project ID ALMA\#2013.1.00226.S, PI: D. K. Öberg). The latter were taken in relatively unstable conditions for the phase and were shifted with respect to our observations. Therefore, the data sets were first individually self-calibrated before being merged, allowing us to properly shift the Cycle 2 observations (via the use of a "false" model point) at the same coordinate position as our data.

We use the Common Astronomy Software Applications (McMullin et al. 2007) software version 4.7.2 for data reduction, self-calibration, and continuum subtraction and version 5.1.1 for deconvolution and imaging. To improve the signal-to-noise ratio, we use a natural cleaning. The resulting synthesized beams for each molecule are given in Table 1 , and the spectral resolution is $0.2 \mathrm{~km} \mathrm{~s}^{-1}$.

\section{Results and Analysis}

\subsection{Emission Maps and Velocity Structure}

The $\mathrm{CO},{ }^{13} \mathrm{CO}, \mathrm{C}^{18} \mathrm{O}$, and $\mathrm{DCO}^{+}$integrated emission maps over the line profile and the $\mathrm{CO}$ velocity map (which is consistent with Keplerian rotation) are displayed in Figure 1. The $\mathrm{CO}$ emission extends beyond that of $1.3 \mathrm{~mm}$ dust continuum (i.e., $r \geqslant 200 \mathrm{au}$ ), as previously observed by Huang et al. (2016). The asymmetry seen in the CO emission map is due to the fact that part of the emission is absorbed by the cloud (see Öberg et al. 2011).

One notable feature shown in Figure 1 is that the spatial distribution of both the $\mathrm{CO}$ and ${ }^{13} \mathrm{CO}$ emission is centrally peaked while that of $\mathrm{C}^{18} \mathrm{O}$ and $\mathrm{DCO}^{+}$emission presents rings. Indeed, $\mathrm{C}^{18} \mathrm{O}$ displays both a centrally bright emission inside the inner $\sim 50$ au of the disk and an outer ring located at $R>120$ au just after the outer continuum gap reported in
Paper I. Regarding the $\mathrm{DCO}^{+}$emission, two rings are observed: the first one located between the two dust continuum gaps (i.e., $66 \mathrm{au} \lesssim R \lesssim 95 \mathrm{au}$ ), and the second one lying after the outer continuum gap at $R>120$ au.

Figures 8-11, in the Appendix, display the $\mathrm{CO},{ }^{13} \mathrm{CO}, \mathrm{C}^{18} \mathrm{O}$, and $\mathrm{DCO}^{+}$channel emission maps, respectively. The fainter lines, associated with $\mathrm{C}^{18} \mathrm{O}$ and $\mathrm{DCO}^{+}$, are detected with at least a peak-to-noise ratio $\geqslant 6 \sigma$ in several channels (i.e., more than 10 channels). The pattern of the $\mathrm{CO},{ }^{13} \mathrm{CO}, \mathrm{C}^{18} \mathrm{O}$, and $\mathrm{DCO}^{+}$ emission is consistent with gas in Keplerian rotation motion.

\subsection{Spectra}

Figure 2 displays the spatially integrated $\mathrm{CO},{ }^{13} \mathrm{CO}, \mathrm{C}^{18} \mathrm{O}$, and $\mathrm{DCO}^{+}$spectra extracted from a 200 au (i.e., $~ 11^{\prime \prime} 6$ ) box centered on AS 209. The line profiles are all consistent with the LSR velocity of the source. In addition, all molecular line shapes are consistent with each other, except that of $\mathrm{CO}$, which displays a brighter redshifted emission. The latter is the result of absorption by the ambient cloud of part of the blueshifted $\mathrm{CO}$ emission (see above, as well as Figures 1 and 3).

\subsection{Line Opacity}

The estimate of the molecular surface density is strongly affected by the opacity of the targeted transition (e.g., Piétu et al. 2007). Assuming isotopic ratios for the local interstellar medium of ${ }^{16} \mathrm{O} /{ }^{18} \mathrm{O}=557$ and ${ }^{12} \mathrm{C} /{ }^{13} \mathrm{C}=70$ (Wilson 1999), we estimate that the $\mathrm{C}^{18} \mathrm{O}$ emission is optically thin throughout the 200 au disk radii (with $\tau\left(\mathrm{C}^{18} \mathrm{O}\right) \leqslant 0.8$ ) while that of ${ }^{13} \mathrm{CO}$ and $\mathrm{CO}$ is optically thick with $\tau \geqslant 2.5$.

These estimates are consistent with the maximum peak intensity maps (moment 8 ; see further details on mom8 maps in Boehler et al. 2017) for the molecular emission lines that are displayed in Figure 3. More specifically, Figure 3 shows moment 8 maps generated with and without continuum subtraction, and one can see that the emission of $\mathrm{C}^{18} \mathrm{O}$ and $\mathrm{DCO}^{+}$is optically thin while this is not the case for $\mathrm{CO}$ and ${ }^{13} \mathrm{CO}$ emission.

\subsection{Radial Intensity Profiles}

Figure 1 show the azimuthally averaged $1.3 \mathrm{~mm}$ dust continuum radial intensity profile along with those of ${ }^{13} \mathrm{CO}$, $\mathrm{CO}$, and $\mathrm{DCO}^{+}$, which have all been deprojected for the AS 209 disk inclination $\left(\mathrm{PA}=86^{\circ}\right.$ and $i=35^{\circ}$; see Paper I). Regarding CO, the displayed profile was averaged at $\pm 20^{\circ}$ along the major west-east emission axis after deprojecting for the disk inclination due to the source geometry (see above and 

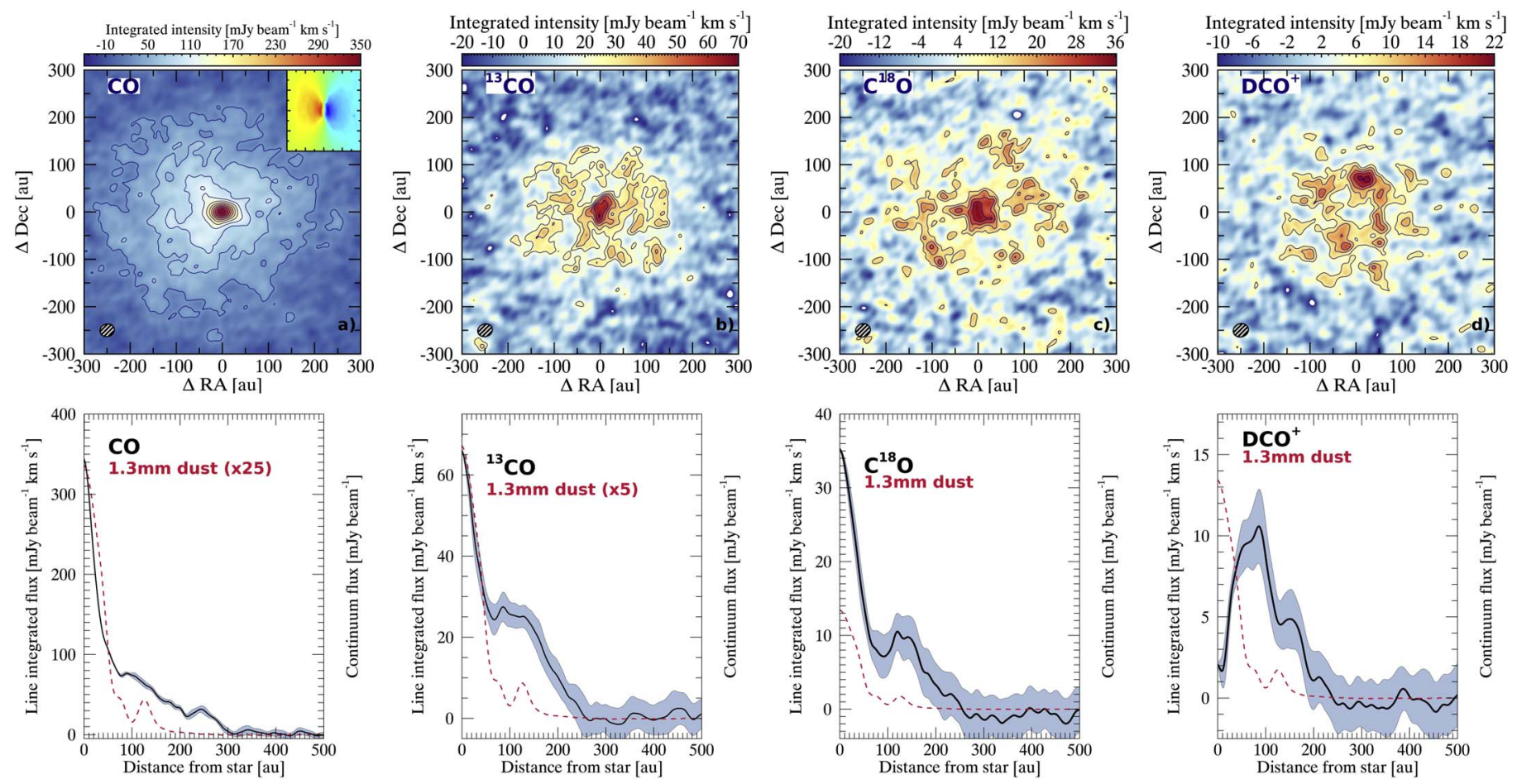

Figure 1. AS 209 emission maps and radial intensity profiles. Top row: (a) CO integrated emission map from $v_{\mathrm{LSR}}=-3.4 \mathrm{up} \mathrm{to} 12.0 \mathrm{~km} \mathrm{~s}{ }^{-1}$. The inset shows the CO intensity-weighted mean velocity map. (b, c) ${ }^{13} \mathrm{CO}$ and $\mathrm{C}^{18} \mathrm{O}$ integrated emission maps from $v_{\mathrm{LSR}}=-1.6$ up to $11.6 \mathrm{~km} \mathrm{~s}^{-1}$. (d) $\mathrm{DCO}^{+}$integrated emission map from $v_{\mathrm{LSR}}=1.8$ up to $7.8 \mathrm{~km} \mathrm{~s}^{-1}$. The first contour is at $2 \sigma$ and the level step is at $1 \sigma$ for $\mathrm{DCO}^{+}$and $\mathrm{C}^{18} \mathrm{O}$ (where $1 \sigma=3.5$ and $6.2 \mathrm{mJy} \mathrm{beam}^{-1} \mathrm{~km} \mathrm{~s}^{-1}$, respectively), while for ${ }^{13} \mathrm{CO}$ and $\mathrm{CO}$ the first contour is at $3 \sigma$ and the level step is at $1 \sigma$ and $3 \sigma$, respectively (where $1 \sigma=8.4$ and $10 \mathrm{mJy} \mathrm{beam}^{-1} \mathrm{~km} \mathrm{~s}^{-1}$ for ${ }^{13} \mathrm{CO}$ and $\mathrm{CO}$, respectively). The synthesized beam is shown in the lower left corner of each panel. Bottom row, from left to right: continuum-subtracted $\mathrm{CO},{ }^{13} \mathrm{CO}, \mathrm{C}^{18} \mathrm{O}$, and $\mathrm{DCO}{ }^{+}$ radial intensity line emission profiles (black line) overlaid with that of the continuum (dashed red line). On each plot, the black line shows the mean profile, while the shaded region shows the standard deviation along the azimuth angle. After deprojecting for the AS 209 disk inclination $\left(i=35^{\circ}\right.$; see Paper I), all the profiles were azimuthally averaged $\left(\mathrm{PA}=86^{\circ}\right.$ ), except that of $\mathrm{CO}$, which was averaged on a wedge $\pm 20^{\circ}$ along the major emission axis.

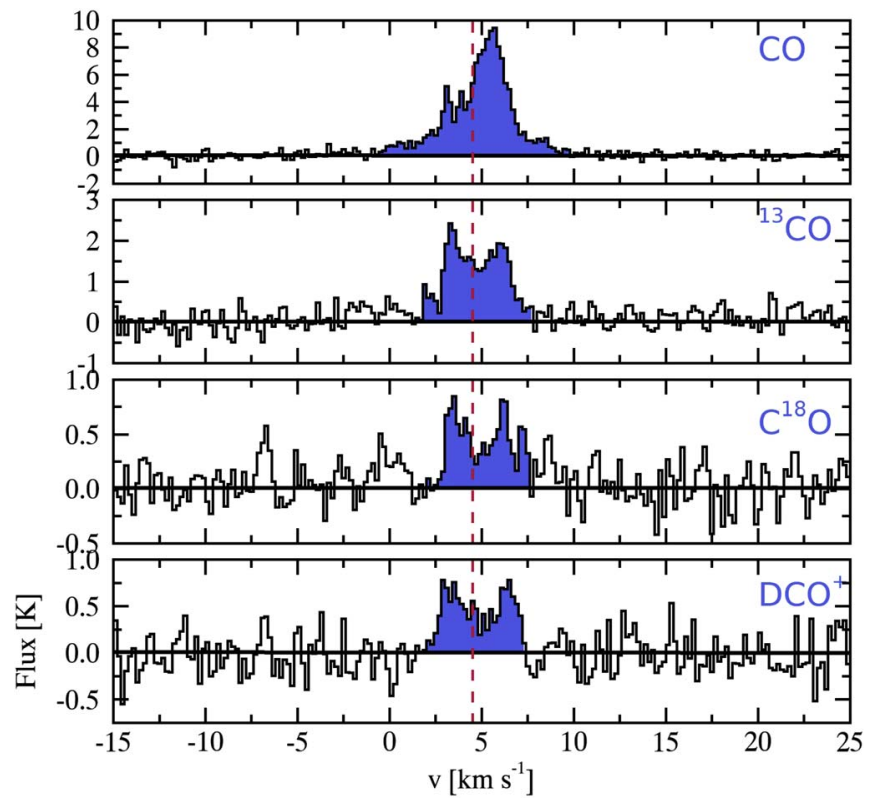

Figure 2. AS 209 disk-averaged spectrum extracted within 200 au for CO, ${ }^{13} \mathrm{CO}, \mathrm{C}^{18} \mathrm{O}$, and $\mathrm{DCO}^{+}$. The vertical red dashed line indicates the LSR systemic velocity, $v_{\mathrm{LSR}} \simeq 4.5 \mathrm{~km} \mathrm{~s}^{-1}$, of the source.

also Teague et al. 2018b) and in order to reduce the noise in the radial profile. It is apparent that the $\mathrm{CO}$ and ${ }^{13} \mathrm{CO}$ emission decreases with increasing radius, which is consistent with previous observations by Huang et al. (2016). The opacity of both lines prevents us from seeing further details.
One notable feature of Figure 1 is that the $\mathrm{C}^{18} \mathrm{O}$ radial intensity profile follows that of the dust continuum and harbors a drop in intensity between the two continuum gaps. Another salient result is that the $\mathrm{DCO}^{+}$radial intensity profile is clearly anticorrelated with that of $\mathrm{C}^{18} \mathrm{O}$ and continuum: while the $\mathrm{DCO}^{+}$intensity increases or decreases with increasing radius, that of $\mathrm{C}^{18} \mathrm{O}$ shows the opposite trend. In particular, the $\mathrm{DCO}^{+}$ surface density is seen between the two continuum gaps.

Finally, Figure 4 displays the AS $209 \mathrm{CO}, \mathrm{C}^{18} \mathrm{O}$, and $\mathrm{DCO}^{+}$ radial profiles along with that of the $1.3 \mathrm{~mm}$ continuum emission and the $1.6 \mu \mathrm{m}$ scattered light radial profile as observed in $H$ band with SPHERE on the Very Large Telescope (VLT; see Avenhaus et al. 2018). As noted by Teague et al. (2018b), the radial locations of both the dust rings and emission peaks are offset between the two grain populations, and the $\mathrm{CO}$ emission peak at about $250 \mathrm{au}$ coincides with that of the $1.6 \mu \mathrm{m}$ scattered light. Incidentally, it is interesting to note that both molecular and micron scattered light extend beyond the millimeter continuum emission.

\section{Gas Gap Thermochemical Modeling}

In this section we aim to investigate whether the deficit of the $\mathrm{C}^{18} \mathrm{O}$ emission is due to perturbations of the gas surface density. As we aim not to perfectly fit the disk temperature and density structures but rather to analyze how gas gaps can affect the molecular emission, we have only performed a simple parametric study of the gas perturbation as described below. 

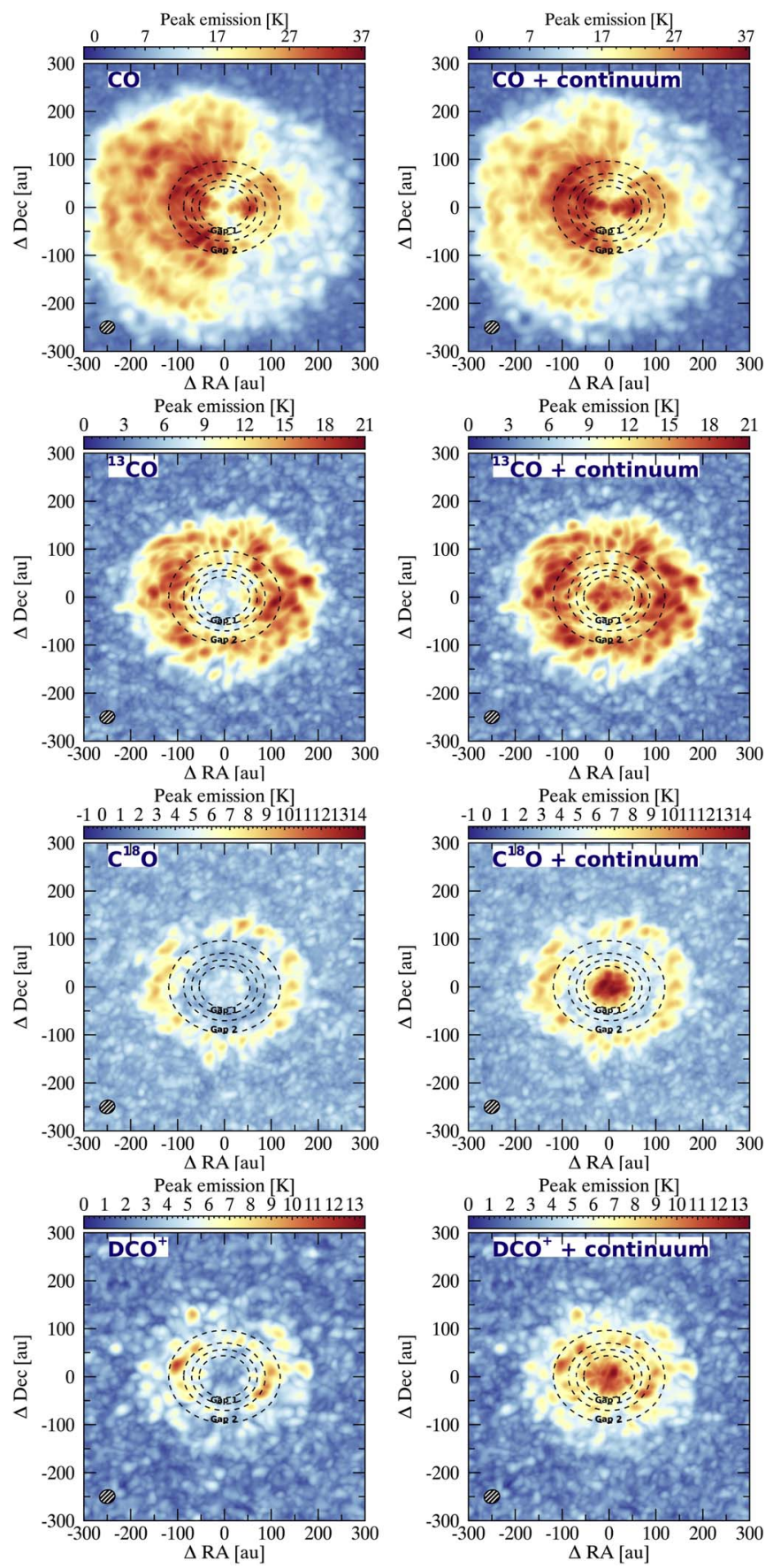

Figure 3. Left column: AS 209 maximum peak intensity emission maps in units of the brightness temperature, for $\mathrm{CO},{ }^{13} \mathrm{CO}, \mathrm{C}^{18} \mathrm{O}(2-1)$, and $\mathrm{DCO}^{+}$ (3-2) emission lines from continuum-subtracted data. Right column: same as the left column, but from native data without continuum subtraction. All the maps are overlaid with the two continuum gaps (black dashed lines) reported in Paper I.

\subsection{Model Description}

We used the thermochemical disk model Dust And LInes (DALI; see Bruderer et al. 2012; Bruderer 2013, for a complete description), which includes the following updates: (i) $\mathrm{CO}$ isotope-selective photodissociation (Miotello et al. 2014, 2016), to reproduce the CO isotopologue emission maps shown in Figure 1, and (ii) density scaling factors to reproduce the AS 209 dust and gas gaps, as described below.

The starting point is the best-fit model of the dust presented in Paper I, which reproduces the $1.3 \mathrm{~mm}$ continuum emission.



Figure 4. Top panel: normalized radial intensity profile of the $\mathrm{CO}$ (yellow) continuum-subtracted emission. The normalized $\mathrm{r}^{2}$-scaled, azimuthally averaged surface brightness of the $1.6 \mu \mathrm{m}$ scattered light (VLT/SPHERE $H$ band, adapted from Avenhaus et al. 2018) is displayed in green color. Bottom panel: normalized radial intensity profiles of the continuum emission at $216 \mathrm{GHz}$ (black) along with that of the $\mathrm{C}^{18} \mathrm{O}$ (red) and $\mathrm{DCO}^{+}$(blue) continuumsubtracted emission (see also Figure 1).

For the dust surface density profile we used that of Paper I. For simplicity, the surface density profile of the small-grain population is assumed to follow that of the large grains (for further details see Section 4.1 of Paper I). The gas density surface density, $\Sigma_{\text {gas }}$, is defined as follows:

$$
\Sigma_{\text {gas }}(R)=\delta(R) \Sigma_{\mathrm{c}, \mathrm{gas}}\left(\frac{R}{R_{\mathrm{c}, \text { gas }}}\right)^{\gamma_{\mathrm{gas}}} \exp \left[-\left(\frac{R}{R_{\mathrm{c}, \mathrm{gas}}}\right)^{2-\gamma_{\mathrm{gas}}}\right]
$$

where $R_{\mathrm{c}, \mathrm{gas}}$ is the cutoff radius of the gas, $\gamma_{\mathrm{gas}}$ is the surface density power-law exponent, and $\delta(R)$ is the surface density scaling factor within the gaps described as follows (by definition $\delta(R)>0)$ :

$$
\delta(R)=\left\{\begin{array}{l}
\delta_{\text {gap }} \text { for } R \in[54 \mathrm{au}, 70 \mathrm{au}] \&[87 \mathrm{au}, 119 \mathrm{au}] \\
\delta_{\text {ring }} \text { for } R \in[70 \mathrm{au}, 87 \mathrm{au}] \\
1 \text { otherwise }
\end{array}\right.
$$

We note that owing to the data angular resolution (allowing us to probe only scale $\geqslant 30 \mathrm{au}$ ), we assumed the same $\delta(R)$ in the two gaps.

In order to reproduce both the absolute flux level and the radial profile of the three $\mathrm{CO}$ isotopologue $(J=2-1)$ transitions, we first run a grid of DALI models ${ }^{9}$ varying $\Sigma_{\mathrm{c}, \mathrm{gas}}, \gamma_{\mathrm{gas}}$, and $R_{\mathrm{c}, \mathrm{gas}}$, while keeping fixed the scale height ${ }^{10} h_{c}$ $(0.133)$ and the flaring exponent $\psi(0.10)$ as in Paper I. Table 2 lists the DALI parameter values adopted in the present study.

In a second step, a grid of DALI disk structures has been created by varying $\delta_{\text {gap }}$ and $\delta_{\text {ring }}$ (the values are given in Table 2). The data-model comparison is performed in the image plane: DALI creates synthetic channel maps, which are

\footnotetext{
9 For the total gas mass, we assumed the standard elemental abundances of carbon and oxygen, $1.35 \times 10^{-4}$ and $2.88 \times 10^{-4}$, respectively.

${ }^{10}$ The scale height distribution is described by $h=h_{c}\left(R / R_{c}\right)^{\psi}$ (for further details see Paper I).
} 
Table 2

Adopted DALI Parameters

\begin{tabular}{lcl}
\hline \hline Fixed Parameter & Value & Description \\
\hline$h_{c}$ & 0.133 & Scale height \\
$\psi$ & 0.1 & Flaring exponent \\
$i(\mathrm{deg})$ & $35^{\mathrm{a}}$ & Disk inclination \\
PA (deg) & $86^{\mathrm{a}}$ & Disk position angle \\
$d(\mathrm{pc})$ & $126^{\mathrm{a}, \mathrm{b}}$ & distance of the source \\
$M\left(M_{\odot}\right)$ & $0.9^{\mathrm{a}, \mathrm{c}}$ & Stellar mass \\
$L\left(L_{\odot}\right)$ & $1.5^{\mathrm{a}, \mathrm{c}}$ & Stellar luminosity \\
$M_{\text {gas }}\left(M_{\odot}\right)$ & $3.0 \times 10^{-3}$ & Disk gas mass \\
$M_{\text {dust }}\left(M_{\odot}\right)$ & $3.5 \times 10^{-4}$ & Disk dust mass \\
\hline Adopted Parameter & $\mathrm{Values}$ & Description \\
\hline$\gamma_{\text {gas }}$ & $0.1, \mathbf{0 . 2}, 0.3,0.4,0.5$ & $\Sigma_{\text {gas }}$ exponent \\
$R_{\text {c,gas }}($ au $)$ & $80,100, \mathbf{1 2 0}, 140,160$ & $\Sigma_{\text {gas }}$ cutoff radius \\
$\delta_{\text {gap }}$ & $\mathbf{0 . 1}, 0.2,0.3,0.4$ & $\Sigma_{\text {gas }}$ scaling factor for \\
$\delta_{\text {ring }}$ & $\mathbf{0 . 4 , 0 . 6 , 0 . 8 , 1 . 0}$ & the gaps \\
& & $\Sigma_{\text {gas }}$ scaling factor for \\
& & the ring \\
\hline
\end{tabular}

Notes. We refer to Paper I for the remaining parameters.

a Paper I.

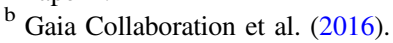

c Andrews et al. (2009).

d The standard elemental abundances of carbon and oxygen, $1.35 \times 10^{-4}$ and $2.88 \times 10^{-4}$, respectively, are assumed.

e The adopted values are given in boldface.

then convolved with a Gaussian beam of the same size as the beam of the observations (see Table 1). Then, the latter are collapsed to create integrated intensity maps.

\subsection{Modeling Results}

Figure 5 shows the surface density parameterization, along with the density and temperature structures of a random DALI model, and the resulting abundance of the three CO isotopologues in the presence of gas gaps. Figure 6 shows the input surface density structure for the different values of

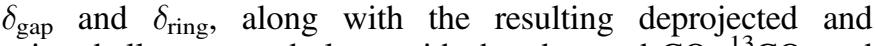
azimuthally averaged along with the observed $\mathrm{CO},{ }^{13} \mathrm{CO}$, and $\mathrm{C}^{18} \mathrm{O}$ radial profiles. The adopted gas density structures reproduce well the overall ${ }^{13} \mathrm{CO}$ and ${ }^{12} \mathrm{CO}$ profiles, although minor differences are seen. The latter are mainly due to the uncertainties in the vertical density and temperature structures (see Bruderer et al. 2012).

Our modeling clearly shows that in order to reproduce the observed $\mathrm{C}^{18} \mathrm{O}$ deficit, $\Sigma_{\text {gas }}$ needs to be lowered, in the gaps, by applying a scaling factor of about $0.1-0.2$ compared to the unperturbed profile (i.e., $\delta(R)=1$ ). Our gas gap thermochemical modeling also shows that the drop of $\mathrm{C}^{18} \mathrm{O}$ is not the result of temperature and/or opacity effects alone (the emission of the $J=2-1$ transition being thin) but that of an intrinsic gas density drop. Indeed, without gas gaps, our model cannot reproduce the $\mathrm{C}^{18} \mathrm{O}$ profile (see Figure 6). Interestingly enough, the ${ }^{13} \mathrm{CO}$ and ${ }^{12} \mathrm{CO} J=2-1$ emission remains optically thick within the gaps. As a consequence, the modeled surface density perturbations lead to minor changes in the resulting ${ }^{13} \mathrm{CO}$ and ${ }^{12} \mathrm{CO}$ radial profiles as shown in Figure 6.

Finally, it is interesting to note that UV irradiation penetrates deeper in the disk layers where gap dusts are located as shown in Figure 5. In that instance, the far-UV radiation field $G_{0}$ is larger and the $\mathrm{C}^{18} \mathrm{O} /{ }^{12} \mathrm{CO}$ mass ratio is further reduced because of photochemistry. From the measured ${ }^{12} \mathrm{CO} / \mathrm{C}^{18} \mathrm{O}$ flux ratio, we estimate that isotope-selective photodissociation (Visser et al. 2009) contributed to about $10 \%-20 \%$ of the observed $\mathrm{C}^{18} \mathrm{O}$ intensity inside the gaps.

We stress that our present modeling does not fully reproduce the observations because of uncertainties on the 2D temperature structure. A more detailed modeling will be presented in an upcoming paper (D. Fedele et al. 2018, in preparation), however.

\section{Discussion}

\subsection{Comparison with Hydrodynamical Simulations}

From our $1.3 \mathrm{~mm}$ continuum observations coupled with hydrodynamical simulations, we have previously shown that the presence of a giant planet or of a pair of planets likely explains the observed dust gaps (see Paper I). Recently, from 2D hydrodynamical simulations of planet-disk interaction, Facchini et al. (2018) have shown that gaps opened by a planet lead to a thermal gas and dust decoupling. The latter strongly affects the dust-to-gas mass ratio within the gaps with respect to the overall ratio. Our DALI modeling of the dust and $\mathrm{C}^{18} \mathrm{O}$ emission is consistent with this interpretation. In addition, hydrodynamical simulations of planet-disk models by Facchini et al. (2018) show that the presence of a giant planet affects not only the dust radial intensity profile but also the ones of $\mathrm{CO}$ and its isotopologues. Indeed, the latter harbor a gap (whose depth and width both depend on the planet mass and disk viscosity; see, e.g., Lin \& Papaloizou 1986; Crida et al. 2006; Fung et al. 2014; Duffell 2015; Dürmann \& Kley 2015; Kanagawa et al. 2017) at the planet location in the disk. In that context, we have thus performed hydrodynamical simulations to further investigate the hypothesis of a planet producing gas gaps in the AS 209 disk. More specifically, we have carried out 2D hydrodynamical simulations using FARGO-3D (see further details in Paper I; Benítez-Llambay \& Masset 2016; Rosotti et al. 2016) for a planet of $0.2 M_{\mathrm{J}}$ and one of $0.3 M_{\mathrm{J}}$ located at $95 \mathrm{au}$. The simulations were run for 1000 orbits of the planet (corresponding to about $10^{6} \mathrm{yr}$ ) and are inviscid (i.e., the viscosity, $\alpha$, is null). The simulations used a grid extending from 19 to $285 \mathrm{au}$, using 1766 logarithmically spaced cells in radius and 4096 cells in azimuth. Wave-killing boundaries were used in the radial direction (de Val-Borro et al. 2006). The disk aspect ratio was taken to be $0.1(R / 100 \mathrm{au})^{0.225}$ as in Paper I. The results are shown in Figure 7 . It is immediately apparent that the presence of a planet perturbes the gas surface density by inducing a gap that is wider and deeper for a massive planet. This is commensurate with our $\mathrm{C}^{18} \mathrm{O}$ observations and the results of Section 4. We infer that the optical thickness of the ${ }^{13} \mathrm{CO}$ and $\mathrm{CO}$ line prevents us from seeing any gap in their radial distribution profiles, as they only probe the disk surface (see Section 4 and Ober et al. 2015). Incidentally, the hydrodynamical simulations show a bump at the location of the planet due to the so-called co-rotation zone. This feature is not seen in our data because we do not have sufficient sensitivity.

\subsection{On the Physical Nature of the $C^{18} \mathrm{O}$ Gap}

Our modeling of the $\mathrm{CO}$ isotopologue emission shows that the drop of $\mathrm{C}^{18} \mathrm{O}$ between the two dust gaps is the result of an intrinsic gas density drop (see Figure 6 and Section 4), which is commensurate with our hydrodynamical simulations. 

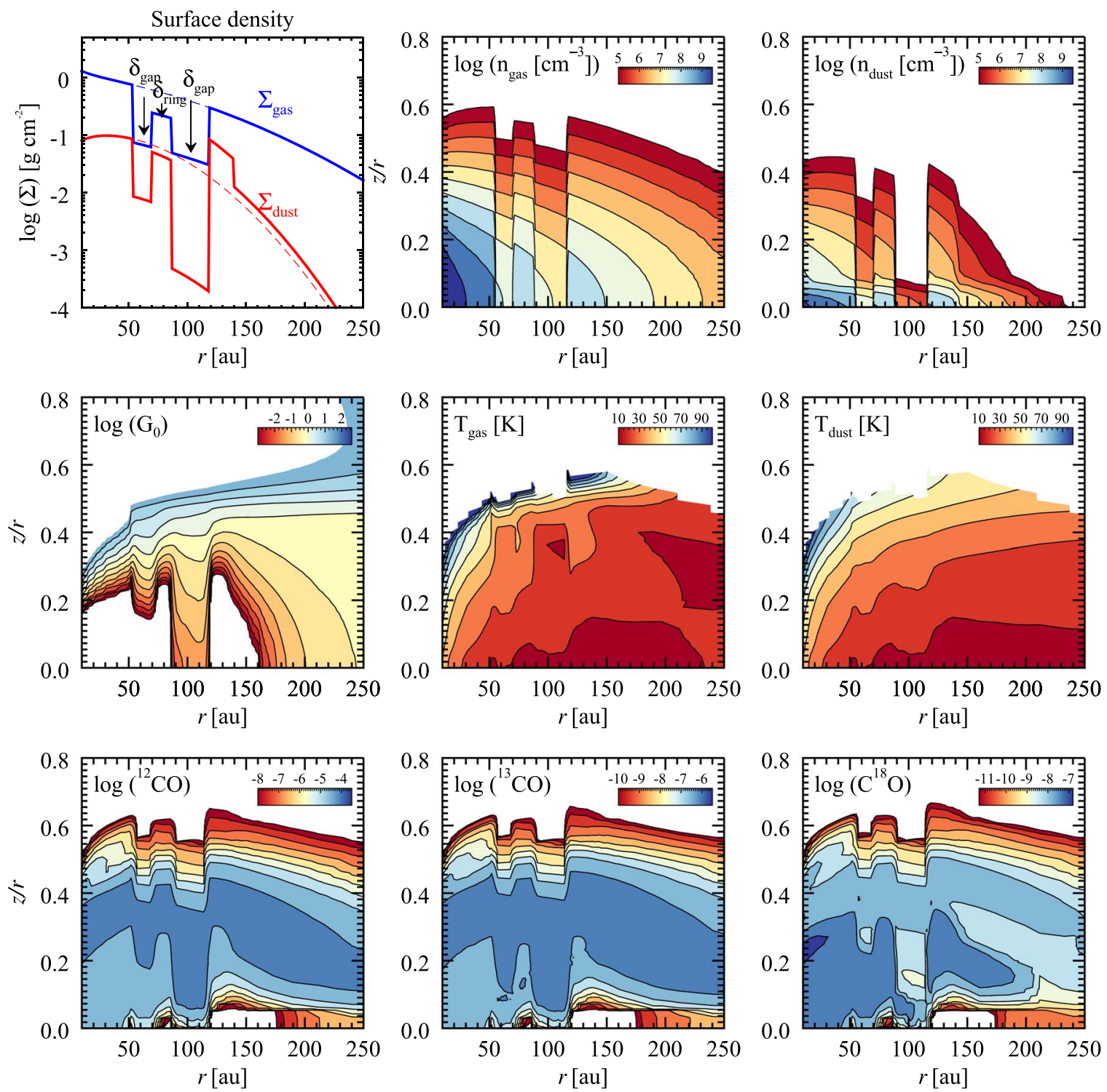

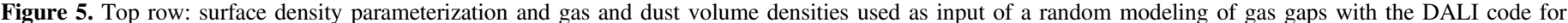

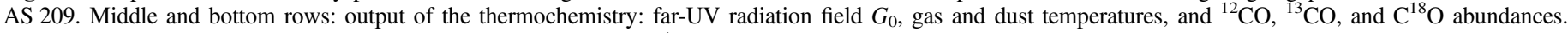
Where not specified, the $y$-axis refers to the vertical scale heights $(z / r)$.

Incidentally, Huang et al. (2016) argued that the observed $\mathrm{C}^{18} \mathrm{O}$ outer ring was due to external photodesorption processes (Facchini et al. 2016). However, the lower angular resolution and sensitivity of their data were not sufficient to allow the authors to see the same structure in the dust emission. Our more sensitive combined high-resolution data clearly show that the outer gas ring seen in $\mathrm{C}^{18} \mathrm{O}$ is also seen in the $1.3 \mathrm{~mm}$ dust emission (see Figures 1 and 4 and Paper I) and, therefore, rule out the hypothesis of external photodesorption.

Our findings strongly support the scenario proposed in Paper I: the formation of a giant planet (at least $M_{\text {planet }}>0.2 M_{\mathrm{J}}$ ) is likely occurring in the AS 209 disk, as (i) the $\mathrm{C}^{18} \mathrm{O}$ outer ring at $120 \mathrm{au}$ is also seen in the $1.3 \mathrm{~mm}$ continuum emission, (ii) the observations can only be reproduced by lowering the gas surface density, and (iii) the wide $\mathrm{C}^{18} \mathrm{O}$ deficiency between the two continuum gaps and the $\mathrm{C}^{18} \mathrm{O}$ increased beyond the dust gaps are both consistent with hydrodynamical simulations of one or more massive planets in formation. Moreover, among the proposed gap-opening mechanisms, magnetorotational instability can only reduce $\Sigma_{\text {gas }}$ by a factor of a few at the edge of the dead zone (Flock et al. 2015), while only a planet-induced dynamical clearing is able to generate the deep gas gaps observed here. Finally, our present data do not allow us to distinguish between the singleplanet scenario that can open multiple gaps for very low viscosity and the one suggesting the presence of two planets (see Paper I; Dong et al. 2015, 2017; Bae et al. 2017).

\section{3. $\mathrm{DCO}^{+}$a Tracer of One or More Forming Planets?}

One notable feature of the $\mathrm{DCO}^{+}$emission toward AS 209 is that it harbors a ring lying in the region located between the two dust continuum gaps (see Figure 1). Interestingly enough, within these dust gaps, one can observe an overdensity of $\mathrm{DCO}^{+}$while neutral gas (i.e., $\mathrm{C}^{18} \mathrm{O}$ ) and dust are depleted (see Figures 1 and 4).

Owing to the low density of both gas and dust within the dust gaps, the medium is likely more ionized than the surrounding regions. Indeed, ionization processes are more 

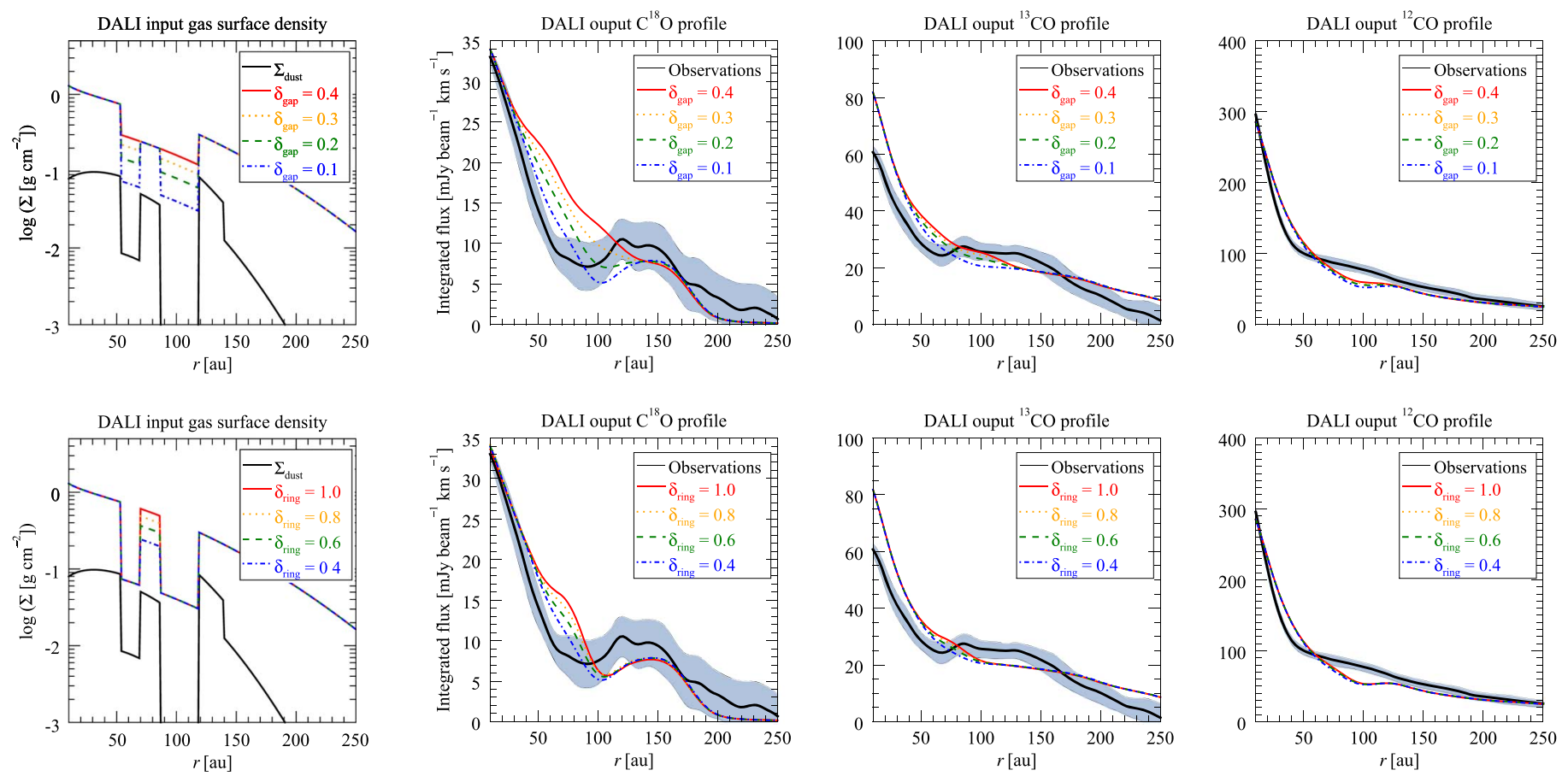

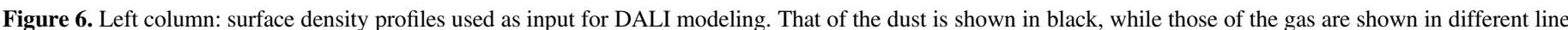

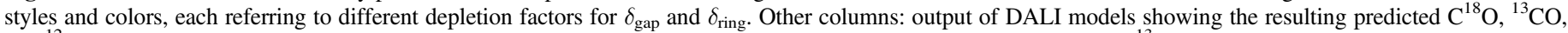

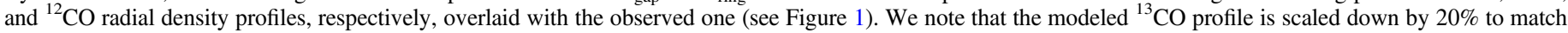
the absolute flux level. Where not specified $\delta_{\text {gap }}=0.1$ and $\delta_{\text {ring }}=0.4$.

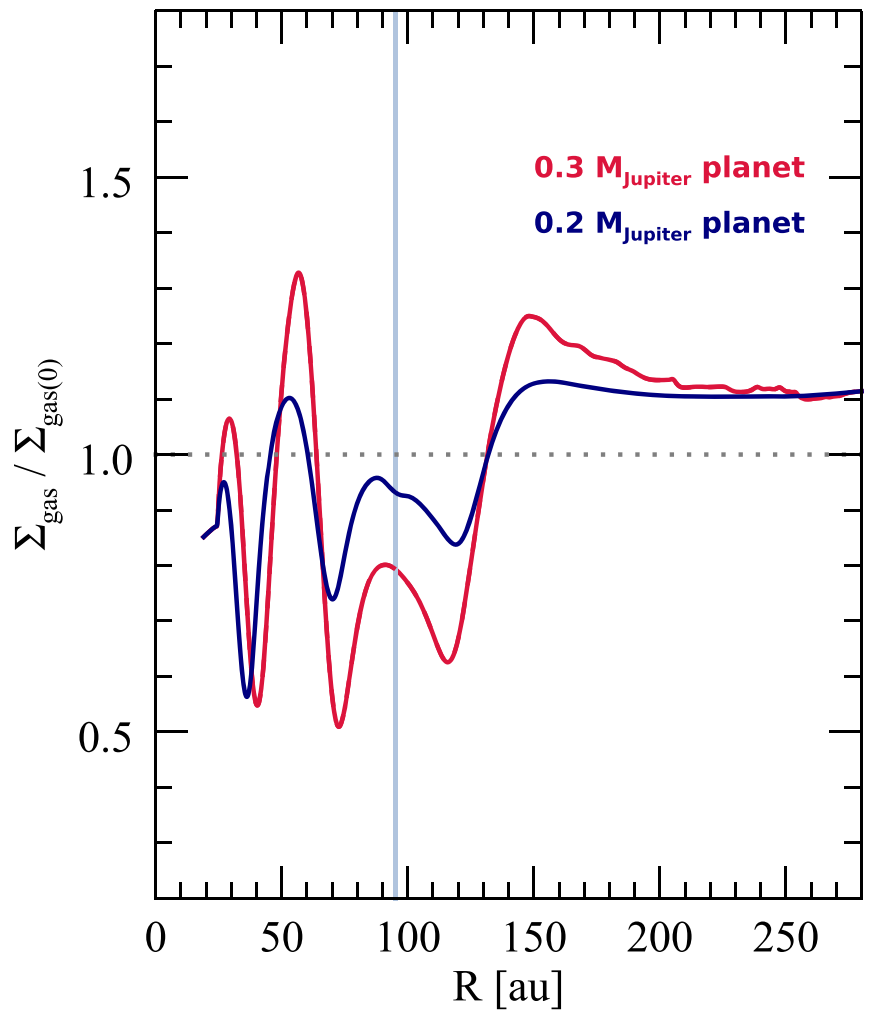

Figure 7. Hydrodynamical simulations of the gas surface density $\left(\Sigma_{\text {gas }}\right)$ perturbed by the presence of a $0.2 M_{\mathrm{J}}$ (blue) planet and a $0.3 M_{\mathrm{J}}$ (red) planet located at $95 \mathrm{au}$. The resulting perturbations are displayed with respect to the unperturbed gas surface density $\left(\Sigma_{\operatorname{gas}(0)}\right)$. The location of the planet is indicated by a vertical line.

efficient in regions of the disk depleted of material, as the gas is less shielded by the dust. Consequently, $\mathrm{DCO}^{+}$enhancement might be the result of multiple chemical pathways such as (i) deuterium exchange between $\mathrm{CO}$ and $\mathrm{H}_{2} \mathrm{D}^{+}$at low temperatures ( $\leqslant 20 \mathrm{~K}$; Watson 1976; Pagani et al. 1992), (ii) that between $\mathrm{HCO}^{+}$and $\mathrm{D}$ atoms (Aikawa et al. 2018), and (iii) that between $\mathrm{CH}_{2} \mathrm{D}^{+}$and $\mathrm{CH}_{4} \mathrm{D}^{+}$at warmer temperatures (30 K $\leqslant T \leqslant 70 \mathrm{~K}$; Favre et al. 2015; Carney et al. 2018), although owing to the distance from the central star and the disk self-shielding in the gap, this region is probably cold. A full physico-chemical modeling of the $\mathrm{DCO}^{+}$chemistry is needed to investigate furthermore the production of $\mathrm{DCO}^{+}$and will be the subject of an upcoming paper. Nonetheless, our finding leads us to suggest that ion enrichment at the location of neutral gas and the dust deficit in protoplanetary disks could be used as a proxy for planet formation.

\section{Conclusions}

Our study shows that the optically thin $\mathrm{C}^{18} \mathrm{O}(2-1)$ and $\mathrm{DCO}^{+}(3-2)$ emission harbors rings and displays anticorrelated radial profiles. More specifically, between the two previously observed dust gaps, there is a deficit of $\mathrm{C}^{18} \mathrm{O}$ while $\mathrm{DCO}^{+}$ (3-2) is enhanced. Our thermochemical modeling of $\mathrm{CO},{ }^{13} \mathrm{CO}$, and $\mathrm{C}^{18} \mathrm{O}$ implies a reduced gas surface density in correspondence with the dust gaps identified in Paper I. These findings led us to infer that the formation of a planet of $0.2 M_{\mathrm{J}}-0.3 M_{\mathrm{J}}$ at about $100 \mathrm{au}$ is occurring in the AS 209 protoplanetary disk. The lower limit of $0.2 M_{\mathrm{J}}$ is needed to reproduce the observed $\mathrm{C}^{18} \mathrm{O}$ deficit between 60 and $110 \mathrm{au}$. The upper limit of $0.3 M_{\mathrm{J}}$ is determined by the size of the dust gaps as measured in Paper I.

An interesting outcome of our thermochemical modeling is that, with $\mathrm{C}^{18} \mathrm{O}$ being more sensitive to the gas and dust density perturbations (in comparison to the optically thicker $\mathrm{CO}$ isotopologues), it is best suited to probe the presence of one or more forming planets down to $\lesssim M_{\text {Saturn }}$ in the outer disk. 
We thank the referee, Dr. Takayuki Muto, for his very fruitful comments that have strengthened our paper. C.F. acknowledges Franck Hersant for fruitful discussion on disk gaps. C.F. and D.F. acknowledge financial support provided by the Italian Ministry of Education, Universities and Research, project SIR (RBSI14ZRHR). A.M. acknowledges an ESO Fellowship. M.T. has been supported by the DISCSIM project, grant agreement 341137, funded by the European Research Council under ERC-2013-ADG. D.S. acknowledges support from the Heidelberg Institute of Theoretical Studies for the project "Chemical Kinetics Models and Visualization Tools: Bridging Biology and Astronomy." The PI acknowledges assistance from Allegro and Bologna, the European ALMA Regional Center nodes in the Netherlands and Italia, respectively. C.F. and D.F. thank J. Huang for sharing the ALMA-Cycle 2 data. This study makes use of ALMA data. ALMA is a partnership of ESO (representing its member states), NSF (USA), and NINS (Japan), together with NRC (Canada), NSC and ASIAA (Taiwan), and KASI (Republic of Korea), in cooperation with the Republic of Chile. The Joint ALMA Observatory is operated by ESO, AUI/NRAO, and NAOJ.

\section{Appendix \\ Channel Emission Maps}

Figures 8-11 display the velocity channel maps for ${ }^{12} \mathrm{CO}$, ${ }^{13} \mathrm{CO}, \mathrm{C}^{18} \mathrm{O}$, and $\mathrm{DCO}^{+}$, respectively.



Figure 8. ${ }^{12} \mathrm{CO}$ velocity channel maps. The first contour and the level step are at $5 \sigma$ (where $1 \sigma=3.5 \mathrm{mJy}$ beam ${ }^{-1}$ ). The synthesized beam is 0 " $25 \times 0$ " $21(\mathrm{PA}$ at $-75^{\circ} .9$ ) 


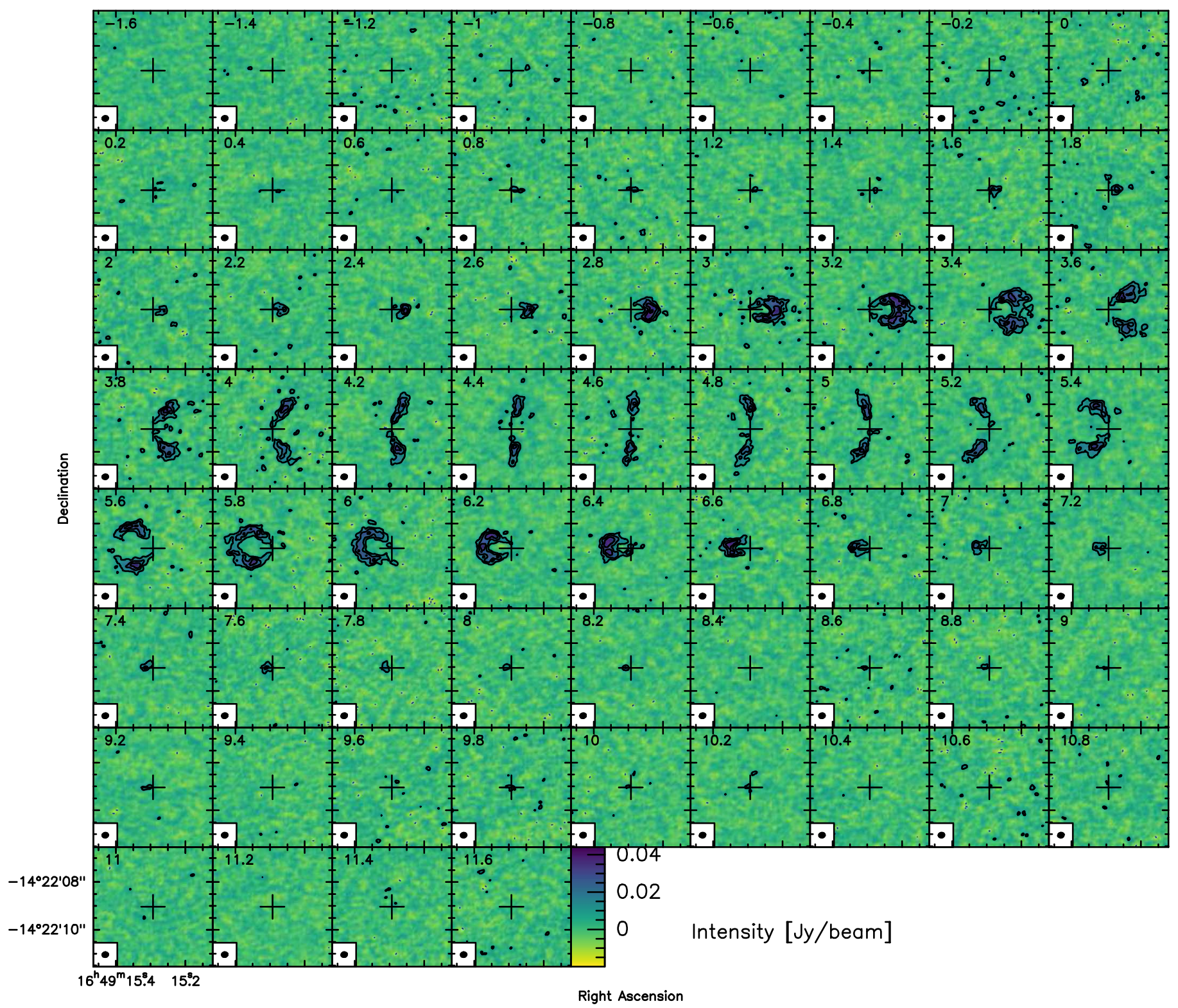

Figure 9. ${ }^{13} \mathrm{CO}$ velocity channel maps. The first contour and the level step are at $3 \sigma$ (where $1 \sigma=3.4 \mathrm{mJy}$ beam ${ }^{-1}$ ). The synthesized beam is 0 " $25 \times 0$.' $21(\mathrm{PA}$ at -73.4 ). 


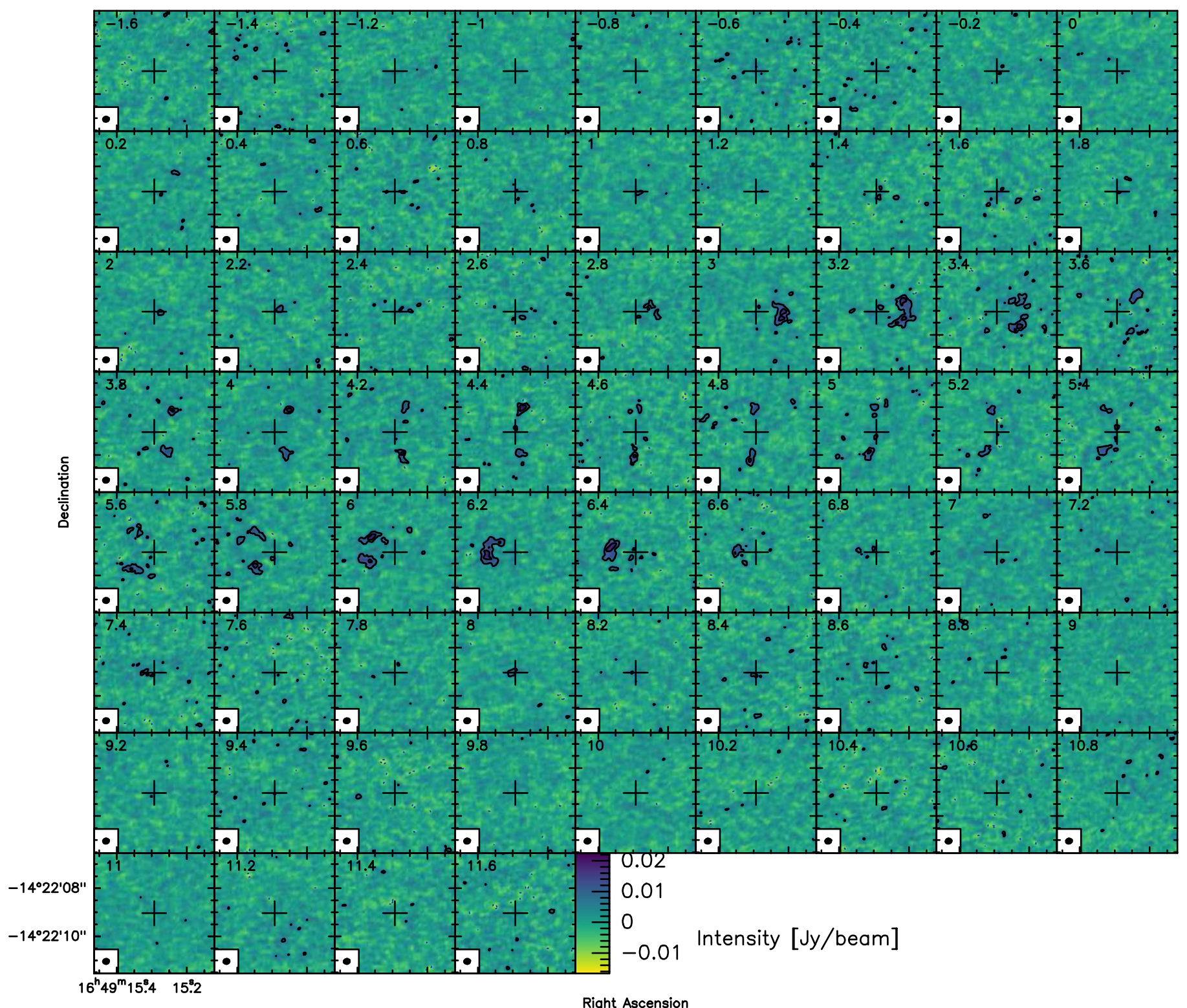

Figure 10. $\mathrm{C}^{18} \mathrm{O}$ velocity channel maps. The first contour and the level step are at $3 \sigma$ (where $1 \sigma=2.6 \mathrm{mJy}$ beam $^{-1}$ ). The synthesized beam is 0 !' $26 \times 0$ !' $22(\mathrm{PA}$ at $-72^{\circ} .4$ ). 


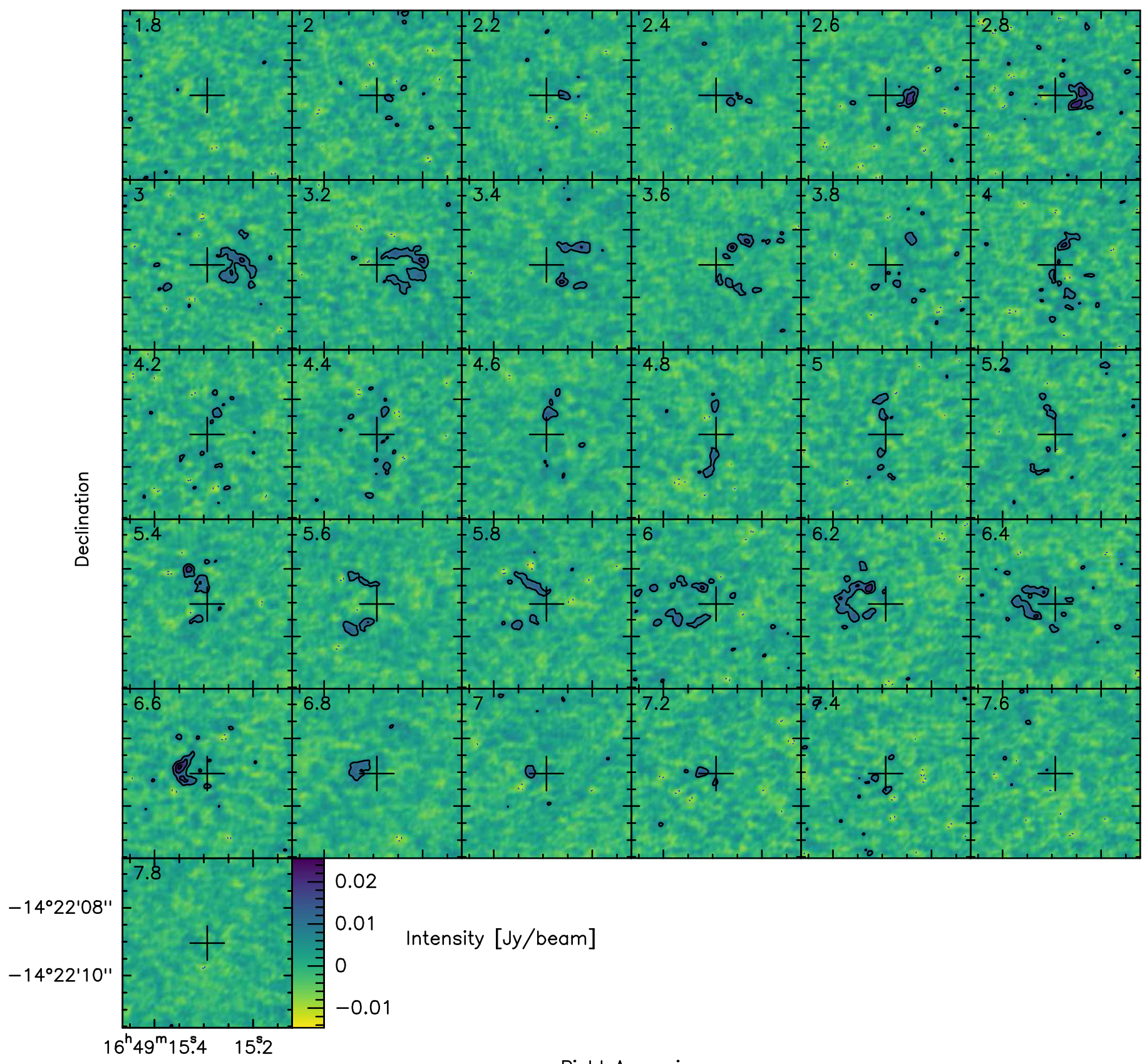

Right Ascension

Figure 11. $\mathrm{DCO}^{+}$velocity channel maps. The first contour and the level step are at $3 \sigma$ (where $1 \sigma=2.6 \mathrm{mJy}$ beam ${ }^{-1}$ ). The synthesized beam is 0 !' $26 \times 0$ !' $21(\mathrm{PA}$ at -73.9 ).

\section{ORCID iDs}

Cécile Favre (iㅏ https://orcid.org/0000-0002-5789-6931 Davide Fedele (1) https://orcid.org/0000-0001-6156-0034 Marco Tazzari (i) https://orcid.org/0000-0003-3590-5814 Leonardo Testi (i) https://orcid.org/0000-0003-1859-3070

\section{References}

Aikawa, Y., Furuya, K., Hincelin, U., \& Herbst, E. 2018, ApJ, 855, 119 ALMA Partnership, Brogan, C. L., Pérez, L. M., et al. 2015, ApJL, 808, L3 Andrews, S. M., Wilner, D. J., Hughes, A. M., Qi, C., \& Dullemond, C. P. 2009, ApJ, 700, 1502

Andrews, S. M., Wilner, D. J., Zhu, Z., et al. 2016, ApJL, 820, L40

Avenhaus, H., Quanz, S. P., Garufi, A., et al. 2018, ApJ, 863, 44
Bae, J., Pinilla, P., \& Birnstiel, T. 2018, ApJL, 864, L26

Bae, J., Zhu, Z., \& Hartmann, L. 2017, ApJ, 850, 201

Benítez-Llambay, P., \& Masset, F. S. 2016, ApJS, 223, 11

Boehler, Y., Weaver, E., Isella, A., et al. 2017, ApJ, 840, 60

Bruderer, S. 2013, A\&A, 559, A46

Bruderer, S., van Dishoeck, E. F., Doty, S. D., \& Herczeg, G. J. 2012, A\&A, 541, A91

Carney, M. T., Fedele, D., Hogerheijde, M. R., et al. 2018, A\&A, 614, A106 Caselli, P., \& Dore, L. 2005, A\&A, 433, 1145

Cazzoli, G., Puzzarini, C., \& Lapinov, A. V. 2004, ApJ, 611, 615

Crida, A., Morbidelli, A., \& Masset, F. 2006, Icar, 181, 587

de Val-Borro, M., Edgar, R. G., Artymowicz, P., et al. 2006, MNRAS, 370,529

Dong, R., Li, S., Chiang, E., \& Li, H. 2017, ApJ, 843, 127

Dong, R., Zhu, Z., \& Whitney, B. 2015, ApJ, 809, 93

Duffell, P. C. 2015, ApJL, 807, L11 
Dürmann, C., \& Kley, W. 2015, A\&A, 574, A52

Facchini, S., Clarke, C. J., \& Bisbas, T. G. 2016, MNRAS, 457, 3593

Facchini, S., Pinilla, P., van Dishoeck, E. F., \& de Juan Ovelar, M. 2018, A\&A, 612, A104

Favre, C., Bergin, E. A., Cleeves, L. I., et al. 2015, ApJL, 802, L23

Fedele, D., Carney, M., Hogerheijde, M. R., et al. 2017, A\&A, 600, A72

Fedele, D., Tazzari, M., Booth, R., et al. 2018, A\&A, 610, A24

Flock, M., Ruge, J. P., Dzyurkevich, N., et al. 2015, A\&A, 574, A68

Fung, J., Shi, J.-M., \& Chiang, E. 2014, ApJ, 782, 88

Gaia Collaboration, Brown, A. G. A., Vallenari, A., et al. 2016, A\&A, 595, A2 Goorvitch, D. 1994, ApJS, 95, 535

Harsono, D., Bjerkeli, P., van der Wiel, M. H. D., et al. 2018, NatAs, 2, 646

Huang, J., Öberg, K. I., \& Andrews, S. M. 2016, ApJL, 823, L18

Huang, J., Öberg, K. I., Qi, C., et al. 2017, ApJ, 835, 231

Isella, A., Guidi, G., Testi, L., et al. 2016, PhRvL, 117, 251101

Kanagawa, K. D., Tanaka, H., Muto, T., \& Tanigawa, T. 2017, PASJ, 69, 97

Klapper, G., Lewen, F., Gendriesch, R., Belov, S. P., \& Winnewisser, G. 2001 ZNatA, 56, 329

Lin, D. N. C., \& Papaloizou, J. 1986, ApJ, 307, 395

Loomis, R. A., Öberg, K. I., Andrews, S. M., \& MacGregor, M. A. 2017, ApJ, 840,23

McMullin, J. P., Waters, B., Schiebel, D., Young, W., \& Golap, K. 2007, in ASP Conf. Ser. 376, Astronomical Data Analysis Software and Systems XVI, ed. R. A. Shaw, F. Hill, \& D. J. Bell (San Francisco, CA: ASP), 127

Miotello, A., Bruderer, S., \& van Dishoeck, E. F. 2014, A\&A, 572, A96
Miotello, A., van Dishoeck, E. F., Kama, M., \& Bruderer, S. 2016, A\&A, 594, A85

Muro-Arena, G. A., Dominik, C., Waters, L. B. F. M., et al. 2018, A\&A, 614, A24

Ober, F., Wolf, S., Uribe, A. L., \& Klahr, H. H. 2015, A\&A, 579, A105

Öberg, K. I., Qi, C., Fogel, J. K. J., et al. 2011, ApJ, 734, 98

Paardekooper, S.-J., \& Mellema, G. 2004, A\&A, 425, L9

Pagani, L., Salez, M., \& Wannier, P. G. 1992, A\&A, 258, 479

Papaloizou, J., \& Lin, D. N. C. 1984, ApJ, 285, 818

Piétu, V., Dutrey, A., \& Guilloteau, S. 2007, A\&A, 467, 163

Pinte, C., Price, D. J., Ménard, F., et al. 2018, ApJL, 860, L13

Rosotti, G. P., Juhasz, A., Booth, R. A., \& Clarke, C. J. 2016, MNRAS, 459, 2790

Teague, R., Bae, J., Bergin, E. A., Birnstiel, T., \& Foreman-Mackey, D. 2018a, ApJL, 860, L12

Teague, R., Bae, J., Birnstiel, T., \& Bergin, E. A. 2018b, ApJ, 868, 113

Teague, R., Semenov, D., Gorti, U., et al. 2017, ApJ, 835, 228

van Boekel, R., Henning, T., Menu, J., et al. 2017, ApJ, 837, 132

Visser, R., van Dishoeck, E. F., \& Black, J. H. 2009, A\&A, 503, 323

Watson, W. D. 1976, RvMP, 48, 513

Wilson, T. L. 1999, RPPh, 62, 143

Winnewisser, G., Belov, S. P., Klaus, T., \& Schieder, R. 1997, JMoSp, 184,468

Winnewisser, M., Winnewisser, B. P., \& Winnewisser, G. 1985, Mol. Astrophys. Ser. C, 157, 375

Zhang, K., Bergin, E. A., Blake, G. A., et al. 2016, ApJL, 818, L16 